\title{
Low-Cost and Robust 1-Gbit/s Plastic Optical Fiber Link Based on Light-Emitting Diode Technology
}

\author{
S.C.J. Lee ${ }^{(1)}$, F. Breyer ${ }^{(2)}$, S. Randel ${ }^{(3)}$, O. Ziemann ${ }^{(4)}$, H.P.A. van den Boom ${ }^{(1)}$, A.M.J. Koonen ${ }^{(1)}$ \\ (1) COBRA Research Institute, Technical University of Eindhoven, P.O. Box 513, 5600 MB, Eindhoven, the Netherlands. (2) Institute for \\ Communications Engineering, Technische Universität München, Arcisstrasse 21, 80290, Munich, Germany. (3) Siemens AG, Corporate \\ Technology, Information \& Communications, Otto-Hahn-Ring 6, 81739, Munich, Germany. (4) POF Application Center, Wassertorstrasse 10, \\ 90489, Nuremberg, Germany.E-mail: s.c.j.lee@tue.nl
}

\begin{abstract}
Gbit/s transmission is demonstrated over $50 \mathrm{~m}$ of step-index PMMA plastic optical fiber (1-mm core-diameter) using a commercial light-emitting diode. This is enabled by use of discrete multitone modulation with up to 64-QAM constellation mapping.

(C)2008 Optical Society of America

OCIS codes: (060.2330) Fiber optics communications; (060.4080) Modulation
\end{abstract}

\section{Introduction}

During the past years, the standard 1-mm core-diameter step-index PMMA plastic optical fiber (SI-POF) has established itself as the preferred transmission medium for robust short-distance data communications in fast-growing markets such as industrial automation networks (PROFINET) and multimedia communication in cars (MOST). Its main benefits are its robustness to electromagnetic interference and mechanical stress, its ease of installation and connection, its low weight, as well as its low price. The latter argument is becoming more and more important as the price of copper has quadrupled from US\$2000 per ton in 2003 to US\$8000 in 2007 [1].

With Intel announcing the development of a new high-speed USB 3.0 standard including optical capabilities [2], optical communications is also finding its way into the consumer market. Nowadays, connector-less SI-POF systems are already being introduced for in-house networks, supporting various applications such as e.g. IP-TV distribution in homes [3]. While today's commercial systems operate at $125 \mathrm{Mbit} / \mathrm{s}$ over up to $100 \mathrm{~m}$ of SI-POF, next generation systems are expected to carry Gigabit Ethernet data over distances of up to $50 \mathrm{~m}$. However, due to its 1-mm large core-diameter and numerical aperture (NA) of 0.5 , the bandwidth of SI-POF is limited to less than $50 \mathrm{MHz} \times 100 \mathrm{~m}$. This makes the possibility of Gigabit transmission over SI-POF seem unlikely. Nevertheless, several advanced modulation techniques have been proposed recently that make this step feasible [4,5]. Especially, by combining multi-carrier modulation with spectrally-efficient quadrature amplitude modulation (QAM), the first demonstration of 1-Gbit/s transmission over $100 \mathrm{~m}$ of SI-POF was reported [4].

An efficient digital implementation of multi-carrier modulation is orthogonal frequency division multiplexing (OFDM) [6], which is already employed in many wireless communications standards such as WLAN, WiMAX, and DVB-T, and is also proposed for next-generation high-bandwidth systems such as Ultra Wideband (UWB). A similar but more cost-effective implementation, discrete multitone (DMT), is widely used in copper-based xDSL systems [7-9]. Considering the industry's extensive experience and the large economies of scale, OFDM and DMT are seen as promising technologies for low-cost, reliable, and robust Gigabit transmission over SI-POF.

So far, all reported Gigabit transmission experiments over SI-POF required the use of laser diodes due to insufficient bandwidth of light-emitting diodes (LEDs). However, LEDs have significant advantages such as high reliability and robustness, longer lifetime, lower cost, less sensitivity to temperature variations, and relaxed eye-safety regulations. Combining such advantages with high-speed Gigabit transmission will make the use of SI-POF even more attractive. In this paper, it is shown that by use of spectrally-efficient DMT modulation, it is possible to realize 1-Gbit/s transmission over $50 \mathrm{~m}$ of SI-POF, even with an LED-based transmitter.

\section{Measurement setup}

Fig. 1 depicts the measurement setup of the DMT transmission system for SI-POF. DMT is a sub-class of OFDM where the output signal from the Inverse Fast-Fourier-Transform is real-valued and no in-phase and quadrature (IQ-) modulation onto an RF-carrier is required [8]. Therefore, broadband, high-frequency, analog RF-components required for IQ-modulation are omitted from DMT-transceivers, reducing system costs and complexity. As a result, only a single digital-to-analog converter (DAC) is needed to create the digitally-generated DMT waveform, which is then used to drive an LED. For the measurement, a DMT time signal consisting of 18000 random DMT symbols is pre-computed using custom software and output cyclically by an arbitrary waveform generator (AWG) with 10 bits of resolution. In order to easily filter out the baseband signal from the high-frequency aliasing products produced by the DAC of the AWG, the 250-MHz DMT waveform is output with 4-times oversampling at a rate of $1 \mathrm{GS} / \mathrm{s}$. In a 


\section{OWB3.pdf}

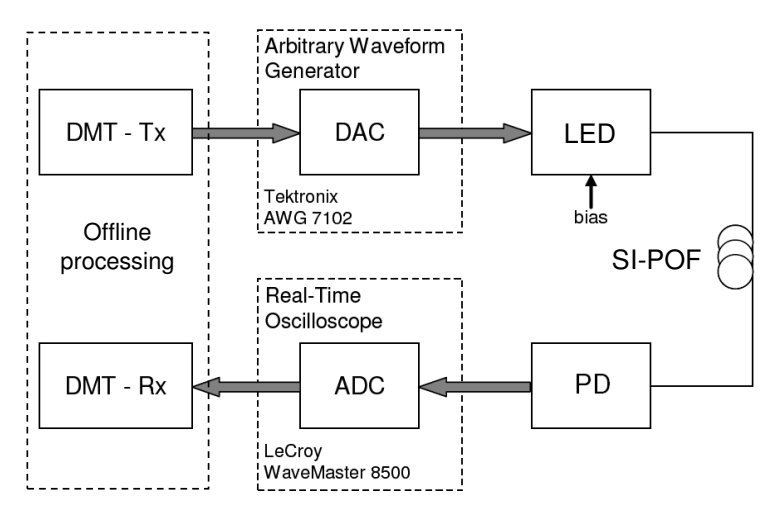

Fig. 1. Measurement setup. Tx: transmitter; Rx: receiver; DAC: digital-toanalog conversion; ADC: analog-to-digital conversion; PD: photodetector.

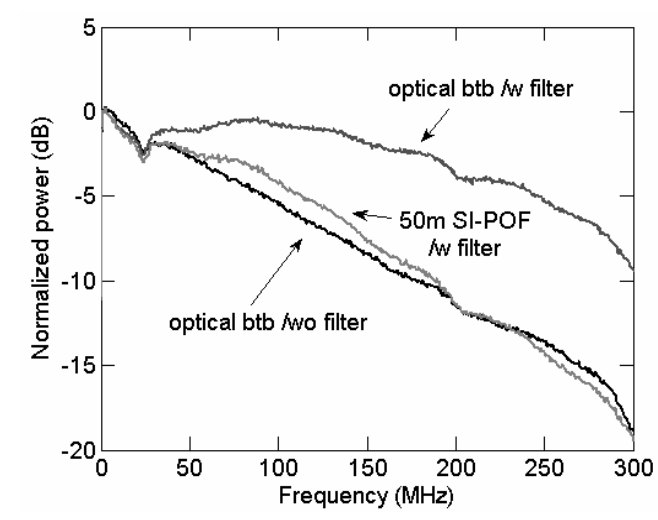

Fig. 2. Measured frequency responses of transmission system with and without passive pre-equalizing filter.

practical system with a properly designed anti-aliasing filter, lower sampling speeds closer to the Nyquist frequency of $500 \mathrm{MS} / \mathrm{s}$ can be used.

The transmitter used in the experiment consists of a commercial resonant-cavity type LED with a wavelength of $650 \mathrm{~nm}$ and an optical output power of $-3 \mathrm{dBm}$, driven in its linear region with a modulation index of approximately 0.8. As can be seen from Fig. 2, the optical back-to-back system has a -3dB bandwidth of around $50 \mathrm{MHz}$, a value which is insufficient for Gigabit transmission using conventional on-off-keying modulation. By adding a passive pre-equalizing filter consisting of a simple analog high-pass filter, the $-3 \mathrm{~dB}$ bandwidth of the optical back-to-back system is increased to about $175 \mathrm{MHz}$. This filter is inserted between the DAC and the LED. Adding $50 \mathrm{~m}$ of commercial SI-POF (6.5dB attenuation, $0.5 \mathrm{NA})$ reduces the bandwidth of the entire system to values similar to the back-to-back case without pre-equalizing filter (see Fig. 2).

After transmission over $50 \mathrm{~m}$ of SI-POF, a $0.8-\mathrm{mm}$ diameter Si photodiode with transimpedance amplifier is used to detect the optical signal. The amplified received electrical signal is then captured by a real-time digital oscilloscope at a sampling rate of $1 \mathrm{GS} / \mathrm{s}$ and a resolution of 8 bits. Using offline processing, the captured DMT data are demodulated and the bit-error ratio (BER) is evaluated.

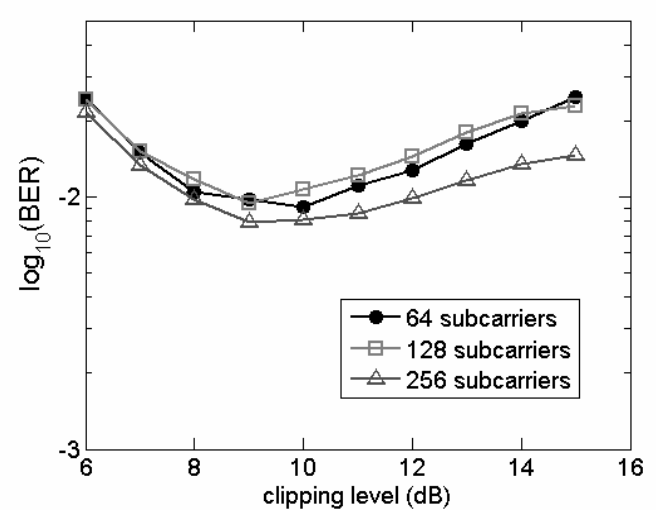

Fig. 3. Measured BER after $50 \mathrm{~m}$ SI-POF for different levels of clipping and number of subcarriers, all with 32-QAM modulation.

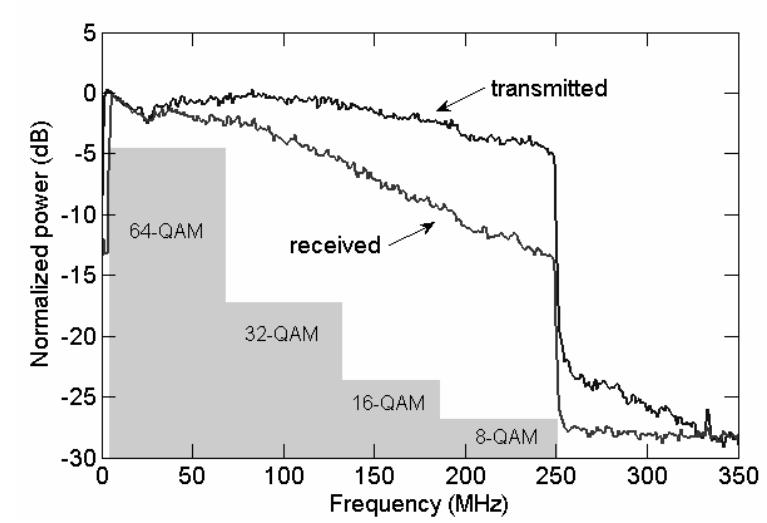

Fig. 4. Transmitted and received (after $50 \mathrm{~m}$ SI-POF) electrical DMT spectra together with applied adaptive QAM-mapping.

\section{Results and discussion}

In multi-carrier transmission systems such as OFDM and DMT, independently modulated subcarriers may incidentally add up constructively, leading to high peak-to-average power ratios in the time signal [6]. In order to utilize the DAC and ADC efficiently, the digitally-generated DMT waveform should be limited in amplitude by clipping. Prior to the transmission experiment, the system including $50 \mathrm{~m}$ of SI-POF is investigated to determine the optimum level of clipping and the optimum number of subcarriers. For this, a DMT signal with $250 \mathrm{MHz}$ of bandwidth and 32-QAM mapping for all subcarriers is used. The cyclic prefix length is kept constant at $3.125 \%$ per DMT symbol, also in the case when a different number of subcarriers are used. Fig. 3 shows the experimental results, indicating that the optimum clipping level is around $9 \mathrm{~dB}$. The clipping level, given in $\mathrm{dB}$, is defined as:

$$
C_{d B}=10 \cdot \log _{10}\left(A^{2} / E\left[|s(t)|^{2}\right]\right),
$$




\section{OWB3.pdf}

where $A$ is the clipped amplitude level and $E\left[|s(t)|^{2}\right]$ denotes the average DMT signal power. From Fig. 3, it can also be seen that for a 50-m SI-POF channel, the use of 256 subcarriers results in the best performance.

These optimum parameters are then used for the transmission experiment. Unlike the previous case, the QAM-mapping per subcarrier is now adapted to the signal-to-noise ratio (SNR) of the transmission channel, as given in Fig. 4. Of the 256 subcarriers, the first five are not used at all because of low SNR. Using this adaptive scheme, a total capacity of 1133 bits per DMT symbol is achieved. This corresponds to a transmit time of $1.024 \mu$ s per DMT symbol, resulting in a total transmission speed of $1133 \mathrm{bits} / 1.024 \mu \mathrm{s}=1106 \mathrm{Mbit} / \mathrm{s}$ within a transmission bandwidth of $250 \mathrm{MHz}$. A spectral efficiency of $4.4 \mathrm{bit} / \mathrm{s} / \mathrm{Hz}$ is hereby achieved.

Fig. 4 shows the transmitted and received electrical DMT signal spectra. Although the $-3 \mathrm{~dB}$ bandwidth of the system with $50 \mathrm{~m}$ of SI-POF is about $50 \mathrm{MHz}, 1-\mathrm{Gbit} / \mathrm{s}$ transmission is realized using DMT. Fig. 5 shows the calculated BER per subcarrier for a total of 18000 received DMT symbols, which equals a total of 20 million bits. Because the serial input data before DMT is transmitted in parallel using subcarriers with DMT, the total BER of the received data should be averaged from all subcarriers used and is calculated to be $4 \cdot 10^{-4}$. By employing forward error correction (FEC) coding, error-free transmission can be achieved. In Fig. 6, the received constellation diagrams after DMT demodulation are depicted, indicating the received signal quality. Every constellation diagram contains the results of all subcarriers with the same constellation. After deduction of $3.125 \%$ of cyclic prefix, $1 \%$ of pilot symbols, and $7 \%$ of FEC overhead, the net transmission bit rate equals $993 \mathrm{Mbit} / \mathrm{s}$.

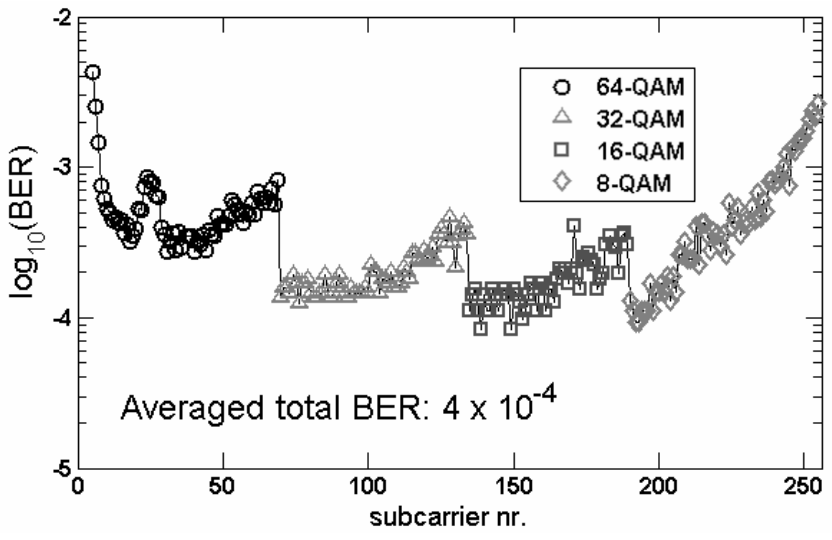

Fig. 5. Calculated BER per subcarrier from measurement results after transmission over $50 \mathrm{~m}$ of SI-POF and DMT demodulation.

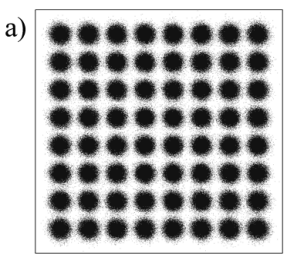

c)

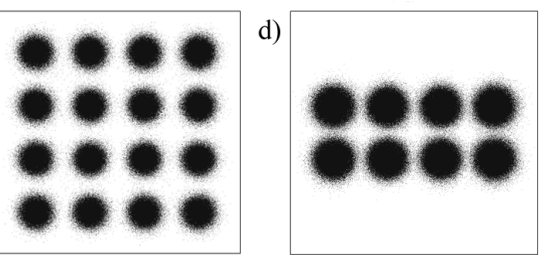

Fig. 6. Overall plot of received constellation diagrams, containing all results from subcarrier nr. (a) 6 to 70 , (c) 71 to 135 , (d) 136 to 190 , (e) 191 to 256.

\section{Conclusions}

1106-Mbit/s transmission is experimentally demonstrated over a 50-m, LED-based, PMMA SI-POF link (-3dB bandwidth of $50 \mathrm{MHz}$ ) using only low-cost, commercially-available components. This is achieved by combining the use of a simple passive high-pass filter and DMT modulation with adaptive QAM-mapping of up to 64-QAM. Building on technologies and hardware for next-generation wireless and wireline systems, DMT proves to be a promising solution for low-cost, robust, and reliable Gigabit transmission over SI-POF based on LEDs.

\section{References}

[1] London Metal Exchange, Copper price [Online]. Available: http://www.lme.co.uk/copper_graphs.asp

[2] Intel Corp. (2007, Sept.). Industry Leaders Develop Superspeed USB Interconnect. [Online]. Available: http://www.usb.org/home

[3] Lightwave Online. (2006, May 17). Infineon unveils POF to Ethernet transceiver reference design for home networking. [Online]. Available: http://lw.pennnet.com/articles/article_display.cfm

[4] S. Randel, S.C.J. Lee, B. Spinnler, F. Breyer, H. Rohde, J. Walewski, A.M.J. Koonen, and A. Kirstädter, "1 Gbit/s Transmission with 6.3 bit/s/Hz Spectral Efficiency in a 100 m Standard 1 mm Step-Index Plastic Optical Fibre Link Using Adaptive Multiple Sub-Carrier Modulation," presented at the Eur. Conf. Optical Commun., Cannes, France, 2006, Postdeadline Paper Th4.4.1.

[5] F. Breyer, S.C.J. Lee, S. Randel, N. Hanik, "1.25 Gbit/s Transmission over up to $100 \mathrm{~m}$ Standard $1 \mathrm{~mm}$ Step-Index Polymer Optical Fibre using FFE or DFE Equalisation schemes", presented at the Eur. Conf. Optical Commun., Berlin, Germany, 2007, paper 9.6.6.

[6] R. van Nee and R. Prasad, OFDM for Wireless Multimedia Communications. Artech House, 2000.

[7] Asymmetric digital subscriber line (ADSL) transceivers, ITU Standard G.992.1, 07-1999.

[8] S.C.J. Lee, F. Breyer, S. Randel, M. Schuster, J. Zeng, F. Huijskens, H.P.A. van den Boom, A.M.J. Koonen, and N. Hanik, "24-Gb/s Transmission over $730 \mathrm{~m}$ of Multimode Fiber by Direct Modulation of an 850-nm VCSEL using Discrete Multi-tone Modulation," presented at the Optical Fiber Commun. Conf., Anaheim, CA, 2007, Paper PDP6.

[9] J. Grubor, S.C.J. Lee, K.D. Langer, T. Koonen, and J. Walewski, "Wireless High-Speed Data Transmission with Phosphorescent White-Light LEDs," presented at the Eur. Conf. Optical Commun., Berlin, Germany, 2007, Postdeadline Paper PD 3.6. 